\title{
Sensitivity of the light field under sea ice to spatially inhomogeneous optical properties and incident light assessed with three-dimensional Monte Carlo radiative transfer simulations
}

\author{
Chris Petrich $^{\mathrm{a}, *}$, Marcel Nicolaus $^{\mathrm{b}}$, Rolf Gradinger ${ }^{\mathrm{c}}$ \\ a Geophysical Institute, University of Alaska Fairbanks, 903 Koyukuk Drive, Fairbanks, Alaska, 99775, USA \\ ${ }^{\mathrm{b}}$ Alfred Wegener Institute for Polar and Marine Research, Bussestrasse 24, 27570 Bremerhaven, Germany \\ c School of Fisheries and Ocean Sciences, University of Alaska Fairbanks, 905 Koyukuk Drive, Fairbanks, Alaska, 99775, USA
}

\section{A R T I C L E I N F O}

\section{Article history:}

Received 25 October 2011

Accepted 21 December 2011

\section{Keywords:}

Sea ice

Optics

Light

Transmission

Monte Carlo

Scattering

\begin{abstract}
A B S T R A C T
Light transmittance through sea ice is affected by surface cover and ice optical properties in the vicinity of the measurement. We present three-dimensional Monte Carlo simulations of light propagation in sea ice to derive upper bounds on the lateral spread of light. Our results give guidance on equipment design and on the possibility of using one-dimensional light transfer models to describe transmittance. Rules were derived for simple cases of optically homogeneous slabs. In the absence of absorption, $10 \%$ and $90 \%$ of the flux detected under optically thick, homogeneous ice are incident on the surface within a radius of less than 0.3 and 2.0 times the ice thickness, respectively. Any increase in optical thickness or absorption in the ice will reduce these radii. For example, the wavelength-dependent absorption of ice results in a $20 \%$ reduction at $700 \mathrm{~nm}$. Optical anisotropy of the slab was also found to produce potentially significant spatial narrowing of the transmitted light field. In the case of direct sunlight, the photon path is displaced toward the sun relative to the location of the detector. This distortion can reach $1 \mathrm{~m}$ or more in optically thick, ponded ice but will be negligible under a surface scattering layer or snow cover. Case studies showed that transmittance of diffuse light in the vicinity of a semi-infinite surface obstruction could be approximated with exponential and error functions. An absorbing cylindrical perturbation of $0.05 \mathrm{~m}$ diameter in $1 \mathrm{~m}$ thick ice placed $1 \mathrm{~m}$ from the point of measurement will absorb less than $1 \%$ of the light otherwise registered by the detector. Many results for transmitted light were independent of the mean cosine of the scattering phase function.
\end{abstract}

(c) 2011 Elsevier B.V. All rights reserved.

\section{Introduction}

Most field measurements of light transmission through sea ice require the ice to be perturbed in order to position a detector. A common measurement method involves drilling an access hole and positioning the instrument either inside or beneath the hole, or beneath the ice at a certain lateral distance from the hole (e.g., Buckley and Trodahl, 1987; Gradinger et al., 2009; Light et al., 2008; Nicolaus et al., 2010a). Using this method, the errors introduced by the physical arrangement have to be estimated. Experiment design will be a trade-off between mechanical constraints of the set-up and errors introduced by the proximity of the detector to any perturbation in the ice or on the surface. Subsequent data analysis is greatly facilitated if the ice above the sensor is horizontally homogeneous as this allows the use of one-dimensional light transfer models. Here

\footnotetext{
* Corresponding author at: Northern Research Institute Narvik, Lodve Langesgate 2, 8514 Narvik, Norway.

E-mail addresses: chris.petrich@gi.alaska.edu (C. Petrich), marcel.nicolaus@awi.de (M. Nicolaus), rrgradinger@alaska.edu (R. Gradinger).
}

the question arises at what spatial range ice should be homogeneous before multi-dimensional approaches should be pursued.

We calculate spatial light propagation through sea ice for generic scenarios in order to determine the order of magnitude of errors listed above. While this cannot replace a careful analysis of the light field following a field campaign, an error estimate will be useful both prior to a campaign for planning purposes and during the campaign. Prior to the campaign, equipment design decisions are made with limited knowledge of actual ice conditions. During a field campaign, experiment design decisions have to be made on the spot. To address both concerns, we seek to derive generic and simple rules for light propagation through sea ice that facilitate informed decision making while detailed case studies are not available. We believe that these practical suggestions are useful for a multitude of field going researchers, interested in physical, biological and geochemical processes within and below the sea ice.

In this study we used a three-dimensional forward Monte Carlo model to simulate light propagation and extinction in a single plane-parallel layer of infinite lateral extent. While sea ice is generally composed of a stack of optically distinct layers of snow, granular ice, and columnar ice, all of which affect the magnitude and spread of 
transmitted irradiance in their own way (see below), we will show that the spatial distribution considered here is reasonably independent of ice type if scaled appropriately. In addition, we will find that the results for transmitted light are reasonably independent of the choice of scattering phase function.

Most simulations of this study were performed on optically isotropic slabs because anisotropic optical properties of sea ice are poorly characterized to-date and because this allowed us to derive an upper estimate of light spread. Unlike air inclusions, brine inclusions in sea ice are usually vertically elongated (Light et al., 2003a), which is thought to cause columnar sea ice to be optically anisotropic. For example, Buckley and Trodahl (1987) reported that they were able to model observed light spread and radiance beneath Antarctic sea ice by treating the granular surface layer as isotropic and the columnar layer as anisotropic with effective scattering coefficients of $5 \mathrm{~m}^{-1}$ and $14 \mathrm{~m}^{-1}$ in vertical and horizontal direction, respectively. Haines et al. (1997) presented similar results for multiple measurement sites and seasons, with ratios between horizontal and vertical scattering coefficient ranging from 1.5 to 2.5. However, in transmittance calculations sea ice is generally treated as optically isotropic (e.g., Grenfell, 1991; Light et al., 2008; Mobley et al., 1998; Perovich, 1990). We will present one example to demonstrate that anisotropy reduces lateral spread noticeably if horizontal scattering coefficients are greater than vertical scattering coefficients. This allows us to derive an upper estimate of beam spread based on isotropic medium calculations. To avoid confusion, note the difference between isotropic scattering, i.e. the direction of a parcel of light after scattering is independent of its incident direction, an optically isotropic medium, i.e. the scattering (and absorption) properties of light are independent of the direction of light, and an isotropic radiation field, i.e. the radiant intensity at a given point is independent of direction.

In sea ice, scattering dominates absorption over much of the visible spectrum in ice layers without substantial concentrations of sea ice sediments and ice algal pigments. Effective scattering coefficients (defined below) between $0.5 \mathrm{~m}^{-1}$ and $150 \mathrm{~m}^{-1}$ have been reported, independent of wavelength but strongly dependent on the type of ice (granular, columnar, meltponds, etc.) (e.g., Light et al., 2008; Mobley et al., 1998; Perovich, 1990). However, spectral absorption of water and ice is strongly dependent on wavelength. In the visible part of the spectrum, it ranges from less than $0.05 \mathrm{~m}^{-1}$ to $0.1 \mathrm{~m}^{-1}$ for wavelengths between 400 and $590 \mathrm{~nm}$ to $0.5 \mathrm{~m}^{-1}$ at $700 \mathrm{~nm}$ (Segelstein, 1981; Warren and Brandt, 2008). In the near-infrared (IR) the absorption coefficient increases exponentially with wavelength, reaching magnitudes of $2 \mathrm{~m}^{-1}$ and $20 \mathrm{~m}^{-1}$ at 800 and $1000 \mathrm{~nm}$, respectively. This renders light in the near-IR almost completely absent under ice. However, light transmitted in the range of photosynthetically active radiation (PAR) from 400 to $700 \mathrm{~nm}$ is of paramount importance for the biological productivity in the Arctic Ocean and biological processes beneath sea ice (Gradinger, 2009). Considering the dominance of scattering in this range, we will perform the majority of the analysis for conservative scattering (i.e., absorption coefficient $\kappa=0$ ), representative for light below $590 \mathrm{~nm}$, and will investigate the effect of absorption separately thereafter.

In this study we focus on investigating the path of light through sea ice and its dependence on ice thickness. After introducing the methods and presenting validation results, we will show case studies to motivate the following more detailed investigation for conservative scattering and absorption. The results section closes with two applied case studies and a brief note on scattering in optically anisotropic slabs. Results are interpreted in the discussion sections and conclusions are drawn for the design of field campaigns and future studies.

\section{Methods}

Monte-Carlo simulations of light propagation through sea ice have been reported previously (e.g., Haines et al., 1997; Light et al., 2003b;
Mobley et al., 1998), so we will limit the description to a brief overview of the particulars employed. In this study we considered light propagation in a homogeneous, plane-parallel slab of thickness $H(\mathrm{~m})$ with scattering and absorption coefficients $\sigma\left(\mathrm{m}^{-1}\right)$ and $\kappa\left(\mathrm{m}^{-1}\right)$, respectively (all symbols used are summarized in Table 1 ). Both $\sigma$ and $\kappa$ are microscopic measures of the probability of scattering and absorption on a straight-line traverse of a parcel of light. They can be expressed in terms of the microscopic extinction coefficient, $k=\sigma+\kappa$, and single-scattering albedo $\omega_{0}=\sigma /(\sigma+\kappa)$, where $k$ is the density of microscopic interactions, and $\omega_{0}$ is the probability that any interaction is a scattering event rather than absorption. Note that these parameters describe a single occurrence of scattering or absorption. Multiple scattering of light is the norm in sea ice, leading to macroscopic, i.e. observable, extinction coefficients and albedo that are considerably different (e.g., Maffione et al., 1998; Pegau and Zaneveld, 2000). The optical thickness, or optical depth, of a slab is a dimensionless quantity defined as $\tau=H(\sigma+\kappa)$.

The Monte Carlo method is a conceptually simple and computationally expensive method of determining radiance inside and at the boundaries of a scattering medium. In this method, discrete parcels of light traverse the medium in straight trajectories between scattering events. The distance between events is determined by a random number generator returning exponentially distributed lengths with specified mean $\delta_{M C}$ (we use subscript MC in this section to label parameters and results of the Monte Carlo algorithm). Scattering events change the direction of travel according to a probability distribution defined by a phase function, $p$. While absorption can be treated as discrete absorption events, the trajectories themselves are independent of absorption allowing the processes of scattering and absorption to be separated. The Monte Carlo model is used to determine the evolution of direction and position of a parcel, while the Beer-Lambert law is invoked at the end of a simulation to account for absorption based on the total path length of the parcel, $D$.

Table 1

List of symbols. Hyphen indicates dimensionless variables.

\begin{tabular}{lll}
\hline Symbol & Unit & Meaning \\
\hline$D$ & $\mathrm{~m}$ & Path length \\
$E$ & $\mathrm{~W} \mathrm{~m}^{-2}$ & Irradiance, spatially \\
$F$ & $\mathrm{~W} \mathrm{~m}{ }^{-2}$ & Irradiance, average \\
$H$ & $\mathrm{~m}$ & Slab thickness \\
$L$ & $\mathrm{~W} \mathrm{~m}^{-2} \mathrm{sr}^{-1}$ & Radiance \\
$N$ & - & Number of repetitions \\
$O$ & - & Position of origin \\
$R$ & $\mathrm{~m}$ & Radial distance from vertical axis through origin \\
$T$ & - & Transmittance \\
$a$ & $\mathrm{~m}$ & Fitting parameter \\
$f$ & - & Fraction of flux registered at detector \\
$g$ & - & Asymmetry parameter (i.e., mean cosine) \\
$k$ & $\mathrm{~m}$ & Microscopic extinction coefficient \\
$n$ & - & Refractive index of slab and medium beneath \\
$p$ & $\mathrm{sr}^{-1}$ & Phase function \\
$r$ & $\mathrm{~m}$ & Radius of cylinder \\
$x$ & $\mathrm{~m}$ & Position in $x$-direction (horizontal) \\
$x_{\mathrm{e}}$ & $\mathrm{m}$ & Position of edge \\
$\Delta x$ & $\mathrm{~m}$ & Separation in $x$-direction \\
$y$ & $\mathrm{~m}$ & Position in $y$-direction (horizontal) \\
$z$ & $\mathrm{~m}$ & Position in $z$-direction (vertical) \\
$\Theta$ & $\mathrm{rad}$ & Difference between angles \\
$\Phi_{\mathrm{T}}$ & $\mathrm{W}$ & Flux registered at detector \\
$\delta$ & $\mathrm{m}$ & Distance to scattering/absorption event \\
$\vartheta$ & $\mathrm{rad}$ & Polar angle with respect to $z$-axis \\
$\kappa$ & $\mathrm{m}$ & Absorption coefficient \\
$\rho$ & - & Random number $0 \leq \rho<1$ \\
$\sigma$ & $\mathrm{m}^{-1}$ & Scattering coefficient \\
$\tau$ & -1 & Optical thickness \\
$\varphi$ & Azimuth angle with respect to $x$-axis \\
$\omega_{0}$ & Single scattering albedo \\
\hline$(1-g)$ & Effective scattering coefficient \\
\hline & &
\end{tabular}


Absorption was accounted for by setting model domain parameters appropriately and calculating exponential attenuation of a parcel from its path length at the end of a trajectory simulation. There are two options for setting model parameters. Operating the model in non-dimensional coordinates, the natural length scale is $\sigma^{-1}$, i.e. the average distance between scattering events. Computations were performed with non-dimensional average length between interactions, $\delta_{M C}^{*}=\sigma^{-1} \sigma=1$, and non-dimensional slab thickness $H_{M C}^{*}=H \sigma$. The resulting non-dimensional path length of a parcel, $D_{M C}^{*}=D \sigma$, was then used to attenuate the parcel according to exp $\left[\begin{array}{ll}-\kappa_{M C}^{*} & D_{M C}^{*}\end{array}\right]$, where the non-dimensional absorption coefficient is $\kappa_{M C}^{*}=\kappa \sigma^{-1}=\left(1-\omega_{0}\right) \omega_{0}^{-1}$. Alternatively, the model was operated in physical coordinates with $\delta_{\mathrm{MC}}=(\sigma+\kappa)^{-1}$ and slab thickness $H_{\mathrm{MC}}=H$. Once a parcel had traveled to a new place of interaction, the direction of travel was changed (i.e. a scattering event took place) with a probability of $\omega_{0}$ or left unchanged for another leg of randomly generated length with probability $\left(1-\omega_{0}\right)$. The parcel was attenuated according to $\exp [-\kappa D]$ at the end of a trajectory simulation. The two methods are mathematically equivalent and we implemented both as a means of verification.

Buckley and Trodahl (1987) modeled sea ice as anisotropic medium with a scattering coefficient that depends on the polar angle of the path of the parcel, $\vartheta$. The scattering coefficient for parcels traveling in direction $\vartheta$ was defined as

$\sigma=\sigma_{v}+\left(\sigma_{h}-\sigma_{v}\right) \sin \vartheta$

where $\sigma_{\mathrm{v}}$ and $\sigma_{\mathrm{h}}$ are the scattering coefficients for vertically and horizontally traveling parcels, respectively (Haines et al., 1997). Calculations were performed for an optically isotropic slab with $\sigma=\sigma_{\mathrm{h}}=\sigma_{\mathrm{v}}$ throughout this manuscript except where stated explicitly.

The probability density of scattering in any particular direction is described by a probability density function $p\left(\mathrm{sr}^{-1}\right)$, referred to as phase function. The most commonly used phase function for MonteCarlo simulations of sea ice is the one-parameter, axially symmetric Henyey-Greenstein phase function (Light et al., 2008; Maffione et al., 1998; Mobley et al., 1998),

$p(\Theta)=\frac{1}{4 \pi} \frac{1-g^{2}}{\left(1+g^{2}-2 g \cos \theta\right)^{3 / 2}}$,

Table 2

Effective scattering coefficients, $\sigma(1-g)$, and asymmetry parameters, $g$, in previous work. B\&L refers to the CCSM implementation of Briegleb and Light (2007). Values of $g$ assumed for numerical reasons are given in brackets.

\begin{tabular}{|c|c|c|c|}
\hline Material & $\sigma(1-g), \mathrm{m}^{-1}$ & $g$ & Reference \\
\hline Snow (cold) & 800 & $(0)$ & Perovich (1990) \\
\hline Snow (cold) & - & $\begin{array}{l}0.8- \\
0.9\end{array}$ & $\begin{array}{l}\text { Grenfell and } \\
\text { Warren (1999) }\end{array}$ \\
\hline Snow (melting) & 160 & $(0)$ & Perovich (1990) \\
\hline Scattering layer (white ice) & 120 & (0) & Perovich (1990) \\
\hline Surface scattering layer (bare ice) & $\begin{array}{l}\text { 20-150 } \\
\text { [B\&L: 60] }\end{array}$ & $(0.94)$ & Light et al. (2008) \\
\hline Granular ice (cold) & 12.5 & 0.95 & Mobley et al. (1998) \\
\hline Drained surface layer (bare ice) & $\begin{array}{l}2.4-12 \\
{[B \& L: 6]}\end{array}$ & $(0.94)$ & Light et al. (2008) \\
\hline Surface scattering layer (ponded) & 4.2 & $(0.94)$ & $\begin{array}{l}\text { Briegleb and Light } \\
\text { (2007) }\end{array}$ \\
\hline Ice interior (cold) & $3-10$ & 0.5 & Haines et al. (1997) \\
\hline Columnar ice (cold) & 4 & 0.98 & Mobley et al. (1998) \\
\hline Ice interior (white ice) & 2.5 & $(0)$ & Perovich (1990) \\
\hline Blue ice (cold) & 1.8 & $(0)$ & Perovich (1990) \\
\hline Columnar ice (cold) & 1.5 & 0.98 & $\begin{array}{l}\text { Pegau and Zaneveld } \\
\text { (2000) }\end{array}$ \\
\hline Blue ice (ponded) & 1.2 & 0 & Perovich (1990) \\
\hline Ice interior (ponded and bare ice) & $\begin{array}{l}0.5-1.8 \\
{[B \& L: 1.2]}\end{array}$ & $(0.94)$ & Light et al. (2008) \\
\hline
\end{tabular}

which is a function of the angle between incident and emerging trajectory, $\Theta$ (Henyey and Greenstein, 1941). The parameter $g$ is the average cosine of the scattered angle, ranging from $g=-1$ for complete backscatter (i.e. reflection) to $g=+1$ for forward scatter (i.e., in effect no scattering). Typical values for snow and sea ice are around $g=0.9$ and $g=0.98$, respectively (Table 2 ). The shape of the Henyey-Greenstein phase function can be seen in Fig. 1. The Eddington phase function is the Henyey-Greenstein phase function expanded in terms of the Legendre Polynomials in $\cos \Theta$ and truncated after the linear term (Thomas and Stamnes, 1999), i.e.

$p(\Theta)=\frac{1}{4 \pi}(1+3 g \cos \Theta)$.

While limited to $|g| \leq 1 / 3$ to ensure $p \geq 0$, the radiation transfer equation can be solved assuming the Eddington phase function (Chandrasekhar, 1960). With $g=0$, Eq. (3) reduces to the isotropic phase function that conveys no relation between the direction of incident and scattered light. Since the phase function is normalized,

$1=\int_{0}^{2 \pi} \int_{0}^{\pi} p(\Theta) \sin \Theta d \Theta d \varphi$

the mean cosine of the phase function, also referred to as asymmetry parameter or anisotropy parameter, is defined as

$g=\langle\cos \Theta\rangle=\int_{0}^{2 \pi} \int_{0}^{\pi} \cos \Theta p(\Theta) \sin \Theta d \Theta d \varphi$

The mean cosine depends on the shape of inclusions and on the refractive index, and ranges from $g=0.86$ for air inclusions in ice to $g=0.98-0.99$ for brine inclusions in cold sea ice (Light et al., 2004; Mobley et al., 1998). A commonly employed similarity relationship is based on the notion that systems have similar optical properties if they agree in their effective scattering coefficient,

$\sigma_{\text {eff }}=\sigma(1-g)$.

This relationship is part of the exact solution of the two-stream approximation of transmittance for conservative scattering (e.g., Bohren, 1987). It has also been suggested as an approximation in the presence of finite absorption, $\kappa>0$ (Light et al., 2003b; van de Hulst, 1980). We made use of this relationship to compare results

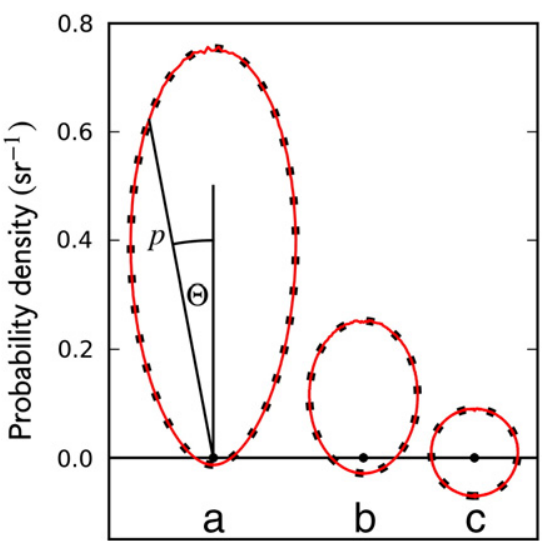

Fig. 1. Polar plots of the probability density of the Henyey-Greenstein phase function for asymmetry parameter (a) $g^{*}=0.59$, (b) 0.35 , and (c) 0.04 (squares), with $\Theta$ plotted as polar angle and phase function $p(\Theta)$ as radius. Lines (simulated data) are the effective phase functions resulting from multiple scattering governed by the HenyeyGreenstein phase function with $g=0.90$ for (a) $N=5$, (b) 10 , and (c) 30 consecutive scatter events. They were derived from the exit angles of $10^{8}$ multiply-scattered parcels binned in intervals of $0.5^{\circ}$. 
between extreme cases of $g=0.98$ and $g=0$. In previous studies, the similarity relationship $\sigma(1-g)$ had been employed to justify the use of smaller asymmetry parameters $g$ to accelerate computations (e.g., Haines et al., 1997; Light et al., 2008). This proved unnecessary for the present calculations. Typical values of the effective scattering parameter $\sigma(1-g)$ range from $0.5 \mathrm{~m}^{-1}$ to $150 \mathrm{~m}^{-1}$ for ponded ice and the surface scatting layer, respectively, and are summarized in Table 2.

Random numbers uniformly distributed in the range $[0 ; 1)$ were generated with the reference implementation of the Mersenne Twister (Matsumoto and Nishimura, 1998). Given a random number $\rho$, an exponentially distributed travel distance for light parcels was derived from $-\delta_{\mathrm{MC}} \ln [1-\rho]$. Upon scattering, the trajectory vector was rotated by a polar angle, $\Theta$, determined from a random number and a precomputed lookup table for the phase function (i.e. the cumulative distribution of the phase function, $p(\Theta)$, weighted with $|\sin \Theta|$ ), and an azimuth angle, $\varphi$, normal to the original trajectory vector and uniformly distributed in $[0 ; 2 \pi)$. Particular care was taken to verify that the expected asymmetry parameter $g$ is produced (Eq. (5)).

We considered two different types of illumination of the slab, direct illumination, i.e. a pencil of light coming from one direction, and diffuse light. While direct illumination represents sunlight under clear skies, diffuse light represents overcast conditions. However, the radiance profile under overcast conditions is not only dependent on cloud thickness, absorption, and solar angle above the clouds, but also on ground albedo (e.g., van de Hulst, 1980). We chose an isotropic radiation field to represent diffuse light under overcast conditions, which is appropriate for optically thick clouds above a reflecting (i.e., optically thick snow or sea ice-covered) ground (van de Hulst, 1980). Similarly, isotropy is also an appropriate assumption for the radiation field at the ice-snow interface if both snow and ice are optically thick (and optically isotropic and conservatively scattering). The refractive index of the slab and the medium beneath the slab was $n$ and may differ from the refractive index above the slab, which was fixed at 1 . For $n>1$, refraction or total reflection took place at the upper surface. Following Light et al. (2003b), all sea ice simulations were performed with $n=1.31$ in the slab and beneath.

In all cases, the light detector was an ideal, infinitesimal small cosine detector with sensor surface parallel to the slab, located at the bottom of the slab at $z=-H$. This detector integrates the energy of all parcels reaching the sensor. The radiant flux transmitted through the slab and registered by the detector is denoted $\Phi_{\mathrm{T}}(\mathrm{W})$. A laterally homogenous irradiance (e.g., irradiance incident at the top of the slab) is denoted $F\left(\mathrm{~W} / \mathrm{m}^{2}\right)$, while laterally varying irradiance (e.g., the areal density of the flux of light parcels reaching the detector) is denoted $E\left(\mathrm{~W} / \mathrm{m}^{2}\right)$.

For the presented simulations the Monte Carlo model was operated in forward mode, i.e. light parcels entered the simulation at the surface of the slab according to the prescribed angular distribution (i.e., diffuse or direct illumination). Upon emergence at the bottom of the slab, the entire light path was laterally shifted to align the point of emergence with the location of the detector. Finally, extinction due to collision of the path with absorbing obstacles was accounted for (i.e., a semi-infinite surface obstruction or an absorbing cylinder in the ice).

\section{Results}

\subsection{Validation}

While results from Monte Carlo simulations are stochastically distributed, the expected accuracy of the results from the Monte Carlo method increases with the number of light parcels. This is illustrated in Fig. 2 for the case of light normally incident on a conservatively scattering slab $(n=1)$ of $H \sigma(1-g)=1$ for isotropic phase function

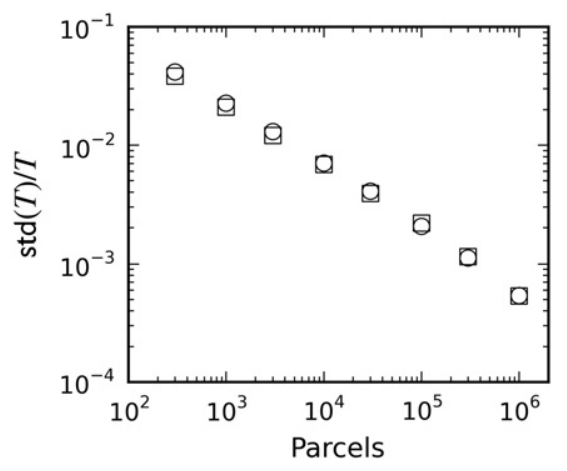

Fig. 2. Standard deviation of transmittance relative to transmittance, $T$, related to the number of parcels normally incident on a conservatively scattering slab $(n=1)$ with optical thickness $H \sigma(1-g)=1$ for Henyey-Greenstein asymmetry parameters $g=0$ (circles) and $g=0.98$ (squares).

$(g=0)$ and Henyey-Greenstein phase function with $g=0.98$. From repeat simulations with a given number of incident parcels, the ratio of standard deviation of the transmittance, $\operatorname{std}(T)$, and transmittance, $T$, was determined. This measure of the uncertainty is related to the number of incident parcels through a power law and is independent of the phase function. The relative uncertainty is below 0.01 for $10^{4}$ parcels. Results in this study were derived from simulations with $5 \times 10^{4}$ to $5 \times 10^{7}$ parcels.

We compared results of the Monte Carlo model with analytical solutions of the radiative transfer equations by Chandrasekhar (1960) for non-refractive slabs. Numerical solutions were tabulated by Chandrasekhar (1960) and Sobouti (1963). Two example calculations are presented of simulated diffuse radiances $L_{d}$ relative to the irradiance normal to the surface of the slab, $F_{0}$. Following Light et al. (2003b), Fig. 3a shows results for a slab of optical thickness $\tau=1$ with $\omega_{0}=1$, isotropic phase function and collimated light incident normal to the surface. Based on 10 repeat simulations, theory and simulations for upwelling and downwelling irradiance agree to within one standard deviation. Fig. 3b illustrates the case of an anisotropic phase functions and absorption. In Fig. 3b the slab is of semi-infinite thickness (approximated numerically by choosing $\tau=100), \omega_{0}=0.8$, Eddington phase function with asymmetry parameter $g=0.33$, and collimated light incident at $37^{\circ}$ elevation angle. The emerging radiance is shown in both forward direction, $\varphi=\varphi_{0} \pm 10^{\circ}$ (positive zenith angles), and backward direction $\varphi=\left(180^{\circ}+\varphi_{0}\right) \pm 10^{\circ}$ (negative zenith angles), and perpendicular to the incident light, at $\varphi=\left(\varphi_{0}+90^{\circ}\right) \pm 5^{\circ}$ and $\varphi=\left(\varphi_{0}-90^{\circ}\right) \pm 5^{\circ}$, respectively. Based on 10 repeat simulations, theory and simulations agree to within one standard deviation.

Van de Hulst (1980) gave tables with numerical results of transmittance and reflectance calculations based on the Henyey-Greenstein phase functions $(0 \leq \mathrm{g} \leq 0.875)$ for different incident light conditions. We verified our implementation of isotropic, incident light (referred to as "narrow source layer" in table 41 of van de Hulst (1980)) by comparing net downward irradiance (i.e., downward irradiance, $F_{\text {down }}\left(\mathrm{W} / \mathrm{m}^{2}\right)$, less upward irradiance $\left.F_{\text {up }}\right)$ at the slab interfaces and in the interior of a slab. An example is shown in Fig. 4 for $\omega_{0}=0.9, g=0.875$, and optical thicknesses spanning three orders of magnitude. The relative error of individual data points is less than $10^{-3}$ or it is of the same order of magnitude as the uncertainty of the tabulated data, whichever is greater.

\subsection{Overview}

The remainder of this section deals with transmitted light parcels terminating at the detector. The coordinate system is illustrated in Fig. 5. We will start with examples for illustration before presenting results in a more general fashion. The following simulations were 
a

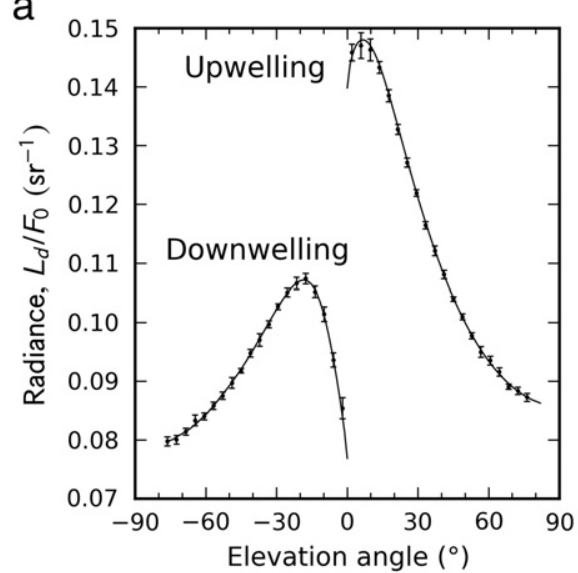

b

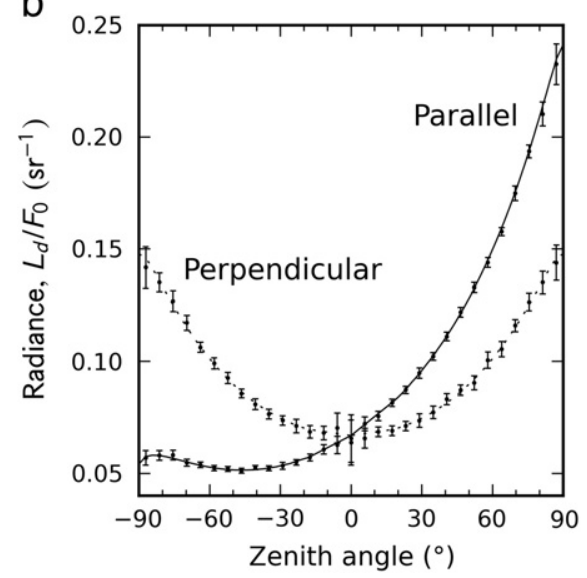

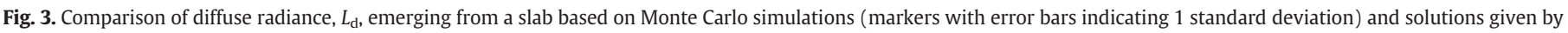

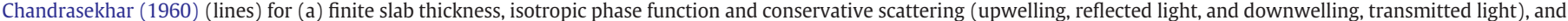

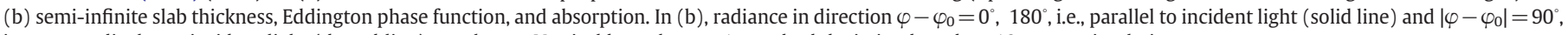
i.e., perpendicular to incident light (dotted line) are shown. Vertical bars show \pm 1 standard deviation based on 10 repeat simulations.

performed with a Henyey-Greenstein phase function with $g=0.98$. Considering a detector at the underside of $1 \mathrm{~m}$ thick ice $(n=1.31)$ with effective scattering coefficient $\sigma(1-g)=2 \mathrm{~m}^{-1}$ (except Fig. 6c, see below) and conservative scattering (except Fig. $6 \mathrm{~d}$, see below), we identified where the flux $\Phi_{\mathrm{T}}$ entered the ice initially with respect to the position of the detector. For the case of diffuse illumination Fig. 6a shows that the distribution of the entrance positions is of radial symmetry around the vertical axis through the detector. The radius within which $f=50,75$, and $90 \%$ of the flux entered is approximately $0.9,1.5$, and $2.1 \mathrm{~m}$, respectively, where

$f=\frac{1}{\pi R^{2} \Phi_{T}} \int_{0}^{2 \pi} \int_{0}^{R} E\left(R^{\prime}, \varphi\right) R^{\prime} d R^{\prime} d \varphi$.

The corresponding areal density of the flux, $E$, is highest within the $50 \%$ perimeter where $E / \Phi_{\mathrm{T}} \geq 0.1 \mathrm{~m}^{-2}$, and decreases exponentially from there on outward, reaching $E / \Phi_{\mathrm{T}}=0.01 \mathrm{~m}^{-2}$ at the $f=90 \%$ perimeter. The same simulation was performed with a pencil of light incident at $23.5^{\circ}$ elevation angle (e.g., solar elevation at noon on 1 April at $71^{\circ} \mathrm{N}$, i.e. during early stages of ice algal bloom), representing direct illumination under clear skies (Fig. $6 \mathrm{~b}$ ). The result is almost the same with the notable difference that the location of entrance is translated $0.3 \mathrm{~m}$ toward the light source, i.e., considerably less than

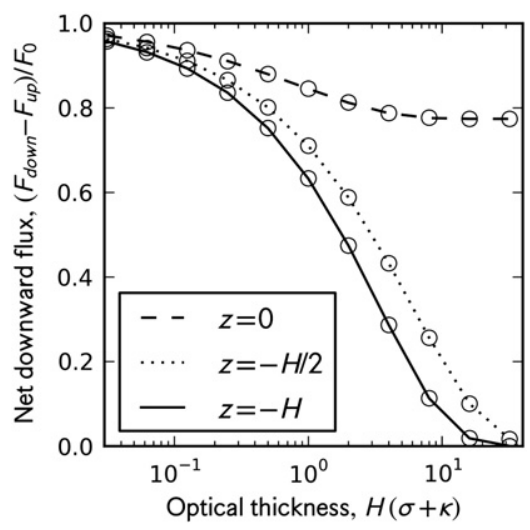

Fig. 4. Net downward irradiance for isotropic illumination of a partially absorbing slab with $\omega_{0}=0.9$ and $g=0.875$. Comparison of model calculations (circles) with tabulated reference data from van de Hulst (1980) for surface (dashed line), center of the slab (dotted line), and bottom (solid line).
$1.0 \mathrm{~m}$ expected from ray optics and refraction following Snell's law. The effect of absorption under direct illumination is shown in Fig. 6d. Compared to the case of conservative scattering in Fig. 6a, absorption in the ice of $\kappa=0.5 \mathrm{~m}^{-1}$ narrows the respective radii considerably to $0.7,1.0$, and $1.5 \mathrm{~m}$, respectively. However, the shift with respect to the detector of $0.3 \mathrm{~m}$ is unaffected. Performing simulations of Fig. 6a for an anisotropically scattering slab, we see that beam spread is reduced to respective radii of $0.7,1.2$, and $1.7 \mathrm{~m}$ (Fig. 6c). The scattering coefficients were chosen such that the transmittance through the slab of Fig. $6 \mathrm{c}$ was equal to the transmittance in Fig. 6a (specifically, $\left.\sigma_{\mathrm{v}}(1-\mathrm{g})=0.87 \mathrm{~m}^{-1}, g=0.98\right)$, and $\sigma_{\mathrm{h}} / \sigma_{\mathrm{v}}=3$ following Buckley and Trodahl (1987).

\subsection{Conservative scattering}

\subsubsection{Direct illumination}

Considering the shift of the irradiance maximum seen in Fig. 6b, we calculated the position of the maximum, $x$, as a function of elevation angle of the incident light for a conservatively scattering medium. Fig. 7 shows that $x$ increases with decreasing solar angle reaching up to approximately $x \sigma(1-g)=0.7$ at low elevation angles

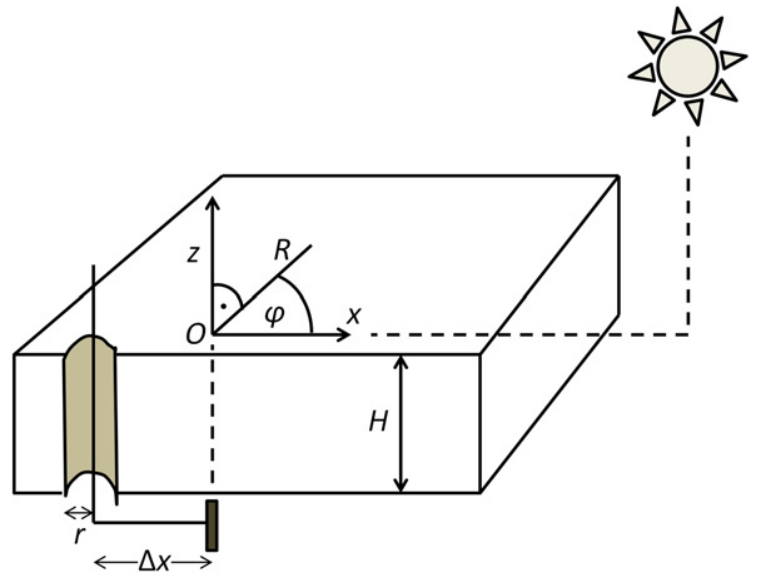

Fig. 5. Illustration of coordinate system used. The origin $O$ is at the surface of the slab of thickness $H$, and the detector is placed at the underside of the slab (dark rectangle), directly beneath the origin (at $z=-H$ ). The source of direct illumination (if applicable) is coming from positive $x$, and an absorbing cylinder (if applicable) of radius $r$ is placed at a distance $\Delta x$ from the detector. The radial component and azimuth angle are denoted $R$ and $\varphi$, respectively. 

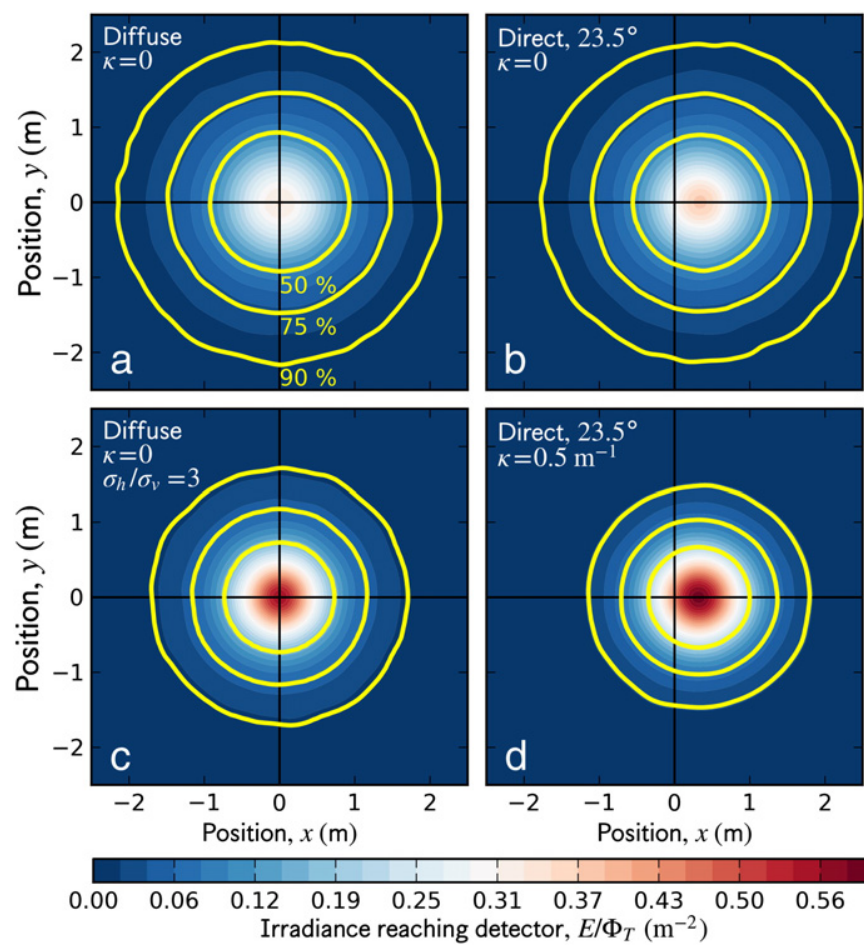

Fig. 6. Surface distribution of the areal density of the flux $\Phi_{\mathrm{T}}$ reaching a detector located beneath the origin of a $1 \mathrm{~m}$-thick slab. Direct illumination at $23.5^{\circ}$ elevation angle (plots b and d) was from positive $x$ toward origin (Fig. 4). Contour lines show the perimeter containing 50,75 , and $90 \%$ of the flux registered at the detector, respectively. The color scale shows the surface areal density of the flux reaching the detector, $E / \Phi_{\mathrm{T}}$. (a) Diffuse illumination in the absence of absorption $(\kappa=0)$, (b) direct illumination in the absence of absorption, (c) diffuse illumination in the absence of absorption, incident on an anisotropically scattering slab with horizontal scattering coefficient, $\sigma_{\mathrm{h}}$, three times as large as the vertical scattering coefficient, $\sigma_{\mathrm{v}}$, and same transmittance as the slab in (a), (d) direct illumination of a slab with absorption coefficient $\kappa=0.5 \mathrm{~m}^{-1}$. The effective scattering coefficient was $\sigma(1-g)=2 \mathrm{~m}^{-1}$ (plots a, b, d) with HenyeyGreenstein phase function and asymmetry parameter $g=0.98$ (all plots).

(for $g=0.98$ ). Calculations were performed for moderately thick ice of $H \sigma(1-g)=1$ and optically thick ice of $H \sigma(1-g)=3$. Within this range, the shift of the maximum, $x$, increases with optical thickness in particular for the isotropic phase function $(g=0$, increase of $50 \%$ at $23.5^{\circ}$ elevation angle), while the sensitivity is less for the strongly forward-peaked Henyey-Greenstein phase function $(g=0.98$,

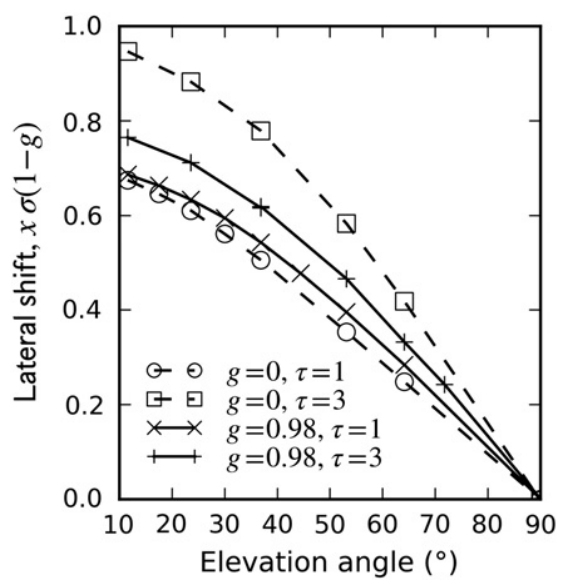

Fig. 7. Shift of the position of maximum flux at the surface with elevation angle of the sun. Medium is conservatively scattering with Henyey-Greenstein phase function with $g=0.98$ (solid lines) and $g=0$ (dashed lines) and optical thickness $\tau=H \sigma(1-g)=1$ (crosses, circles) and $\tau=H \sigma(1-g)=3$ (pluses, squares). Refractive index of the slab is $n=1.31$. increase of $10 \%$ at $23.5^{\circ}$ elevation angle). For $g=0.98, x$ is generally around $x \sigma(1-g)=0.6 \pm 0.1$ for common angles of incidence.

The vertical profiles in Fig. 8 show the average displacement, $x$, of the passage of light parcels that eventually reach the detector under the slab (at $x=0)$. The slab $(n=1.31)$ is conservatively scattering, and illuminated with direct light incident on the slab at $23.5^{\circ}$ elevation angle. Fig. 8a is the vertical profile corresponding to the case of Fig. 6b. For the strongly forward-peaked phase function $(g=0.98$, Fig. 8a) the average position reaches its maximum of 0.4 to 0.5 optical thicknesses at the surface for ice thicknesses $H \sigma(1-g) \geq 1$. For thinner ice $(H \sigma(1-g)=0.5)$, the maximum position is less. In the case of isotropic scattering (Fig. 8b), the displacement near the surface is higher and the maximum is not reached until $H \sigma(1-g)>1$. For optically thin slabs $(H \sigma(1-g)<1)$, the vertical profile of $x$ is linear. Optically thick slabs $(H \sigma(1-g) \geq 3)$ show an exponential profile with non-dimensional $e$-folding length of 2.3 and 1.3 for the HenyeyGreenstein phase function $(g=0.98)$ and isotropic scattering, respectively.

\subsubsection{Diffuse illumination}

Under diffuse light illumination, where the radiance distribution is isotropic, the light field is directionally invariant around the vertical axis through the detector. Corresponding to Fig. 6a, Fig. 9 shows the radii, $R$, of the circles at the surface of the slab that contain $f=50$, 75 , and $90 \%$ of the flux reaching the detector, $\Phi_{\mathrm{T}}$, respectively. The radii, expressed in multiples of ice thickness, $H$, depend on the optical thickness of the slab, $H \sigma(1-g)$. They increase with optical thickness for optically thin ice and decrease, following a power-law relationship, for optically thick ice. While the cases of isotropic scattering and strongly forward-peaked phase function $(g=0.98)$ are indistinguishable for optically thick ice $(H \sigma(1-g) \geq 3)$, significant differences exist for ice thinner than $H \sigma(1-g)=1$. However, the scaled radius of the $50 \%$ contour is nearly independent of optical thickness for optically thin slabs. In Fig. 10, the corresponding vertical profiles of $R$ of the 50 and $90 \%$ contour lines are shown for $H \sigma(1-g)=1$ and 8 . Similar to observations

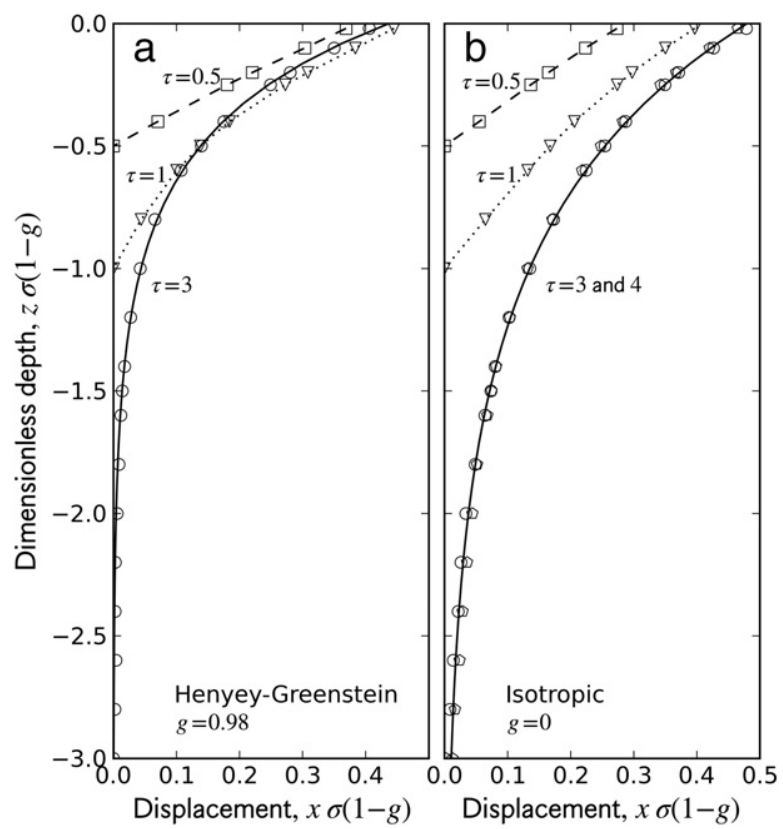

Fig. 8. Displacement, $x$, of the mean position of the passage of parcels toward the light source at planes, $z$, in the ice for non-dimensional ice thicknesses $\tau=H \sigma(1-g)=0.5$ (squares, dashed line), 1.0 (triangles, dotted line), 3.0 (circles), and 4.0 (pentagons, only in (b)). The solid line is an exponential fit through data of $\tau=H \sigma(1-g)=3.0$. Results for (a) Henyey-Greenstein phase function with asymmetry parameter $g=0.98$ and (b) isotropic phase function. Conservative scattering, elevation angle of incident light: $23.5^{\circ}$. Note the different scales on abscissa and ordinate. 


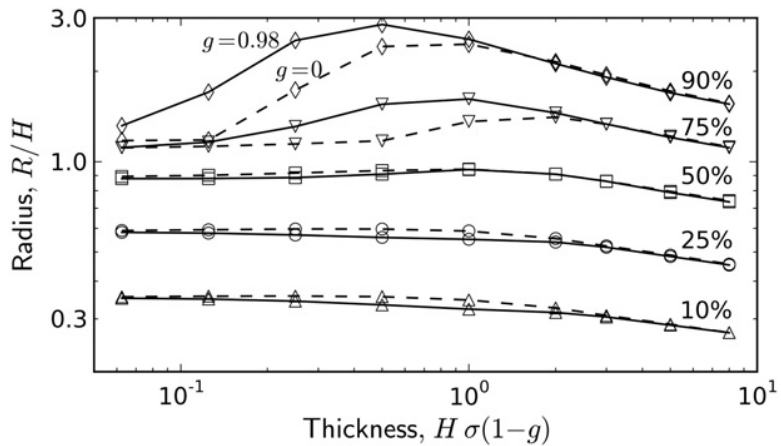

Fig. 9. Radius, $R$, of the circle at the surface encompassing 10\% (upward-pointing triangles), $25 \%$ (circles), $50 \%$ (squares), $75 \%$ (downward-pointing triangles), and $90 \%$ (diamonds) of all parcels reaching the detector beneath the slab of optical thickness $H \sigma(1-g)$, based on conservative scattering with isotropic $(g=0)$ (dashed line) and the Henyey-Greenstein phase function with $g=0.98$ (solid line).

in Fig. 9 for these particular optical thicknesses, no appreciable differences exist between isotropic phase function and $g=0.98$. While the radius of the $50 \%$ contour decreases linearly with depth for $H \sigma(1-g)=1$, all other cases are more bowl-shaped with relatively depthindependent radii in the upper half of the slab.

The radial dependence of the flux $\Phi_{\mathrm{T}}$ reaching the detector is expressed as irradiance, $E$ (i.e., areal flux density), at the surface of the slab. Fig. 11a shows this dependence for optical thicknesses between 0.25 and 8 , and $g=0.98$, corresponding to the color scale in Fig. 6a. For optically thick slabs $(H \sigma(1-g) \geq 3), E$ decreases exponentially with radial distance $R$. The exponential decrease starts at $R /$ $H>1.2$ with an extinction coefficient in $R / H$ of -2.1 to -2.7 for $H \sigma(1-g)=3$ and 8 , respectively. At shorter radial distances, the curves are qualitatively similar but differ in magnitude at $R=0$. The relative irradiance at $R=0$ is highest for the optically thickest slabs. The calculations were repeated with an isotropic phase function and shown in Fig. 11b. While results for $H \sigma(1-g) \geq 3$ agree with the case of $g=0.98$, systematic differences become apparent for optically thinner slabs. Slabs of $H \sigma(1-g) \leq 1$, show a halo-like increase of irradiance as $R / H$ approaches 1.2 from below, where $\tan \left(\arcsin n^{-1}\right)=$ 1.2 , i.e. related to the critical angle of total internal reflection. A discontinuous change in irradiance is observed with substantially lower irradiances on the far side of $R / H=1.2$. A similar distortion is visible for the case of $g=0.98$ in Fig. 11a for $H \sigma(1-g)=0.25$, albeit significantly less pronounced.

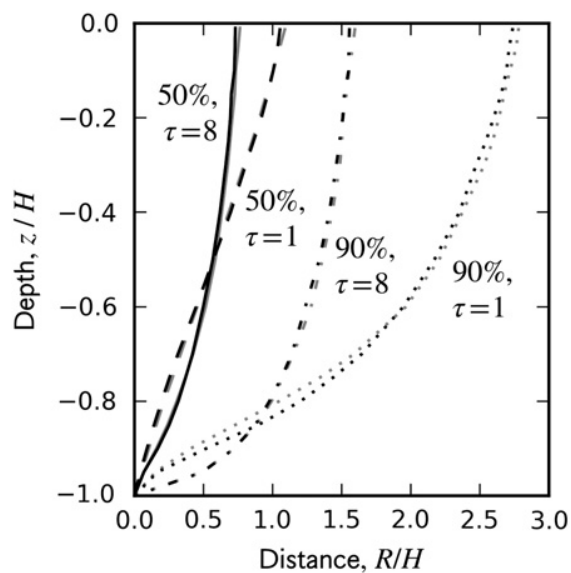

Fig. 10. Depth-dependence of the radius $R$ of the circle encompassing $50 \%$ and $90 \%$ of the passages of packages reaching the detector. Examples given for $\tau=H \sigma(1-g)=1$ (dashed and dotted lines) and 8 (solid and dash-dotted lines) for $50 \%$ (solid and dashed lines) and 90\% (dash-dotted and dotted lines), and for $g=0.98$ (black) and $g=0$ (gray). Scattering is conservative.

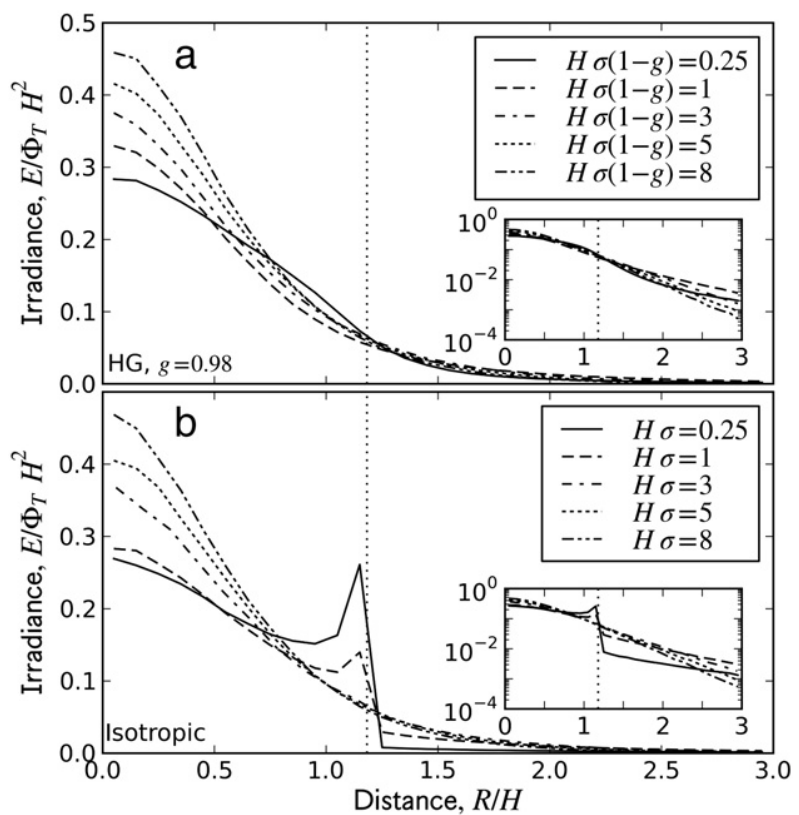

Fig. 11. Incident irradiance (i.e., areal density of the fluxreaching the detector), $E / \Phi_{\mathrm{T}}$, as a function of distance $R$ from the origin. The vertical axis is normalized with slab thickness squared, $H^{2}$. The vertical dotted line indicates $R / H=\tan \left(\arcsin n^{-1}\right)$. Inset shows the same data on semi-logarithmic scale. Slab of thickness $H$ is conservatively scattering with (a) Henyey-Greenstein phase function with asymmetry parameter $g=0.98$ and (b) $g=0$.

\subsection{Absorption}

We assessed the influence of absorption for specific scattering conditions and slab thicknesses. In particular, we used effective scattering coefficients $\sigma(1-g)=0.5$ and $2 \mathrm{~m}^{-1}$ with $g=0.98$, slab thicknesses $H=0.5$ and $1 \mathrm{~m}$, diffuse illumination, and absorption coefficients from $0.05 \mathrm{~m}^{-1}$ to $20 \mathrm{~m}^{-1}$. We evaluated the radii of the circles encompassing $f=50,75$, and $90 \%$ of the flux registered at the detector. Fig. 12 shows the radii $R$ for varying absorption coefficients $\kappa$ in relation to the respective radius for conservative scattering, $R(\kappa=0)$. The spread of the flux at the surface decreases with increasing absorption, reducing to anywhere between 0.8 and 0.4 times their value for conservative scattering at $\kappa=20 \mathrm{~m}^{-1}$. The reduction is more pronounced for a $1 \mathrm{~m}$ thick slab (Fig. 12b) than for a slab of $0.5 \mathrm{~m}$ (Fig. 12a). Within any physical configuration, the reduction is most pronounced at the $90 \%$-percentile. At $\kappa=0.5 \mathrm{~m}^{-1}$, radii have typically decreased to 70 to $80 \%$ of their respective values in the case of conservative scattering.
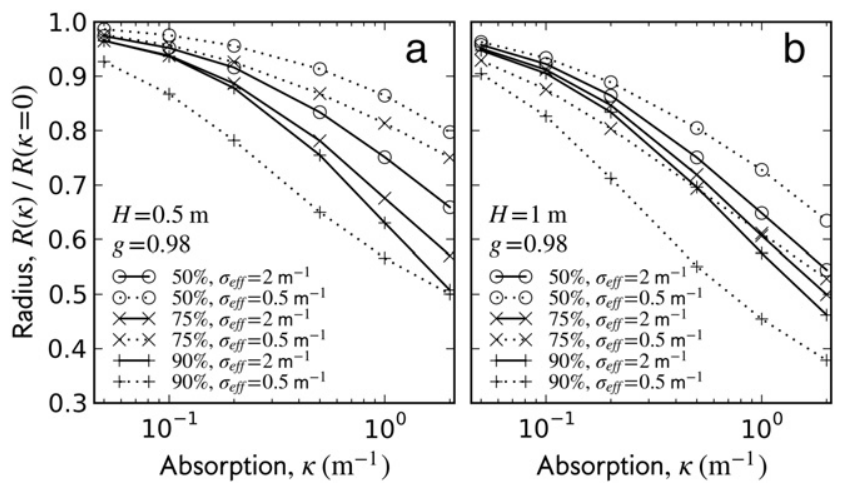

Fig. 12. Influence of absorption coefficient, $\kappa$, on radius, $R$, of percentiles relative to radius under conservative scattering $(\kappa=0)$. Data for $\sigma_{\text {eff }}=\sigma(1-g)=2 \mathrm{~m}^{-1}$ (solid lines) and $\sigma_{\text {eff }}=\sigma(1-g)=0.5 \mathrm{~m}^{-1}$ (dotted lines), percentiles $50 \%$ (circles), $75 \%$ (crosses), and 90\% (pluses), and ice thickness (a) $0.5 \mathrm{~m}$ and (b) $1 \mathrm{~m}$. Simulations are based on Henyey-Greenstein phase function with $g=0.98$. 


\subsection{Optically anisotropic slab}

Simulations were performed for conservative scattering with the Henyey-Greenstein phase function $(g=0.98)$ in an anisotropically scattering slab. In order to compare simulations of light propagation through optically isotropic and anisotropic slabs we had to define an invariant. We chose to require the transmittance of slabs of equal thickness to be invariant. Through successive approximation we found that an isotropic slab of thickness $H=1 \mathrm{~m}$ with $\sigma(1-g)=$ $2 \mathrm{~m}^{-1}$ (Fig. 6a) exhibits the same transmittance as an anisotropically scattering slab of $\sigma_{\mathrm{v}}=(0.87 \pm 0.005) \mathrm{m}^{-1}$ and $\sigma_{\mathrm{h}} / \sigma_{\mathrm{v}}=3$, where the ratio between horizontal and vertical scattering was based on results of Buckley and Trodahl (1987). The results shown in Fig. 6c (described above) show that the lateral spread is reduced. Based on successive approximation we found that vertical scattering coefficient for $\sigma_{\mathrm{h}} / \sigma_{\mathrm{v}}=2$ would be $\sigma_{\mathrm{v}}=(1.19 \pm 0.005) \mathrm{m}^{-1}$.

\subsection{Multiple scattering}

While passing through an optically thick slab, parcels are scattered multiple times before reaching the detector. We investigated the effect of multiple scattering on the effective phase function, i.e. the phase function that would have let to the same intensity distribution after a single scattering event. Starting with the Henyey-Greenstein phase function with $g=0.90$, Fig. 1 compares the effective phase function observed after $N$ consecutive scattering events with a Henyey-Greenstein phase function of modified asymmetry parameter, $g^{*}=g^{N}$. This particular relationship between degree of multiple scattering, $N$, and effective asymmetry parameter, $g^{*}$, is specific to the Henyey-Greenstein phase function and has been described elsewhere (e.g., Piskozub and McKee, 2011; Zege et al., 1991). The effective phase function becomes asymptotically isotropic as a result of multiple scattering.

\subsection{Case studies}

\subsubsection{Semi-infinite obstruction}

Consider a detector fixed at $x=0$ under a $1 \mathrm{~m}$ thick slab with effective scattering coefficient $\sigma(1-g)=2 \mathrm{~m}^{-1}$ and $g=0.98$. Illumination is diffuse. The slab has a semi-infinite surface obstruction with edge at $x_{e}$ that prevents light from entering the slab at $x>x_{e}$. How does the apparent transmittance $T$ depend on the distance between detector position and the edge of the surface obstruction? Fig. 13 shows the transmittance, i.e. the ratio between irradiance $F_{0}$ at the surface and irradiance detected under the slab as a function position of the edge. Calculations are performed for conservative scattering,

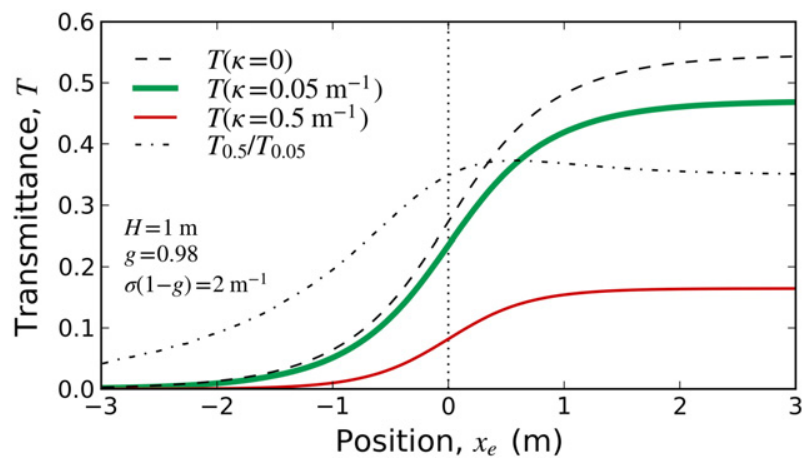

Fig. 13. Transmittance, $T$, observed by a detector in the vicinity of a semi-infinite surface obstruction. The detector is placed at 0 and the position of the edge of the semiinfinite obstruction, $x_{e}$, is plotted on the abscissa. Negative and positive positions refer to the semi-infinite obstruction above and beside the detector, respectively. Conservative scattering (dashed line), absorption $\kappa=0.05 \mathrm{~m}^{-1}$ (thick solid line), $0.5 \mathrm{~m}^{-1}$ (thin solid line), and ratio between transmittance based on $\kappa=0.5$ and $0.05 \mathrm{~m}^{-1}$ (dash-dotted line).

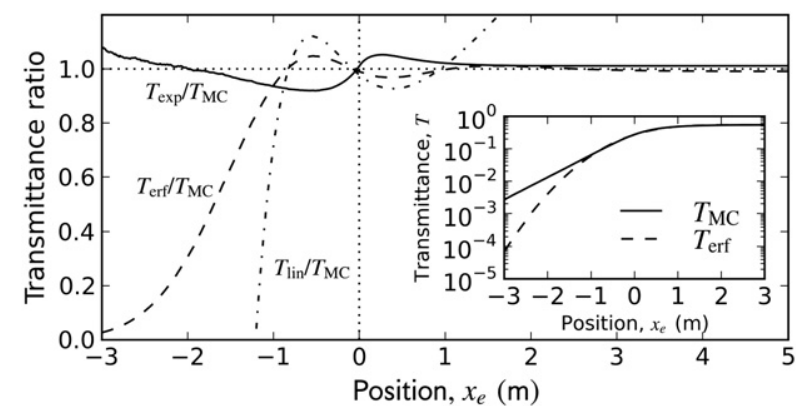

Fig. 14. Comparison of transmittance ratio as function of position of edge and detector between exponential fit ( $T_{\text {exp }}$, solid line), error function ( $T_{\text {erf }}$, dashed line), and linear fit

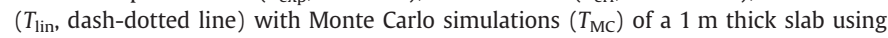
conservative scattering. Inset shows transmittance of Monte Carlo simulations $\left(T_{\mathrm{MC}}\right.$, solid line) and error function ( $T_{\text {erf }}$, dashed line) data in semilogarithmic scale. The detector is placed at 0 and the semi-infinite obstruction is above the detector for negative positions of the edge, $x_{e}$.

and for absorption coefficients $\kappa=0.05 \mathrm{~m}^{-1}$ ("green light") and $0.5 \mathrm{~m}^{-1}$ ("red light"). As the edge of the obstruction is further than $3 \mathrm{~m}$ from the detector $\left(x_{e}>3 \mathrm{~m}\right)$, transmittance has assumed the farfield limit and is independent of edge position. At $x_{e}=0$, i.e. with the edge directly above the detector, apparent transmittance has reduced to half of the far-field limit, and as the obstruction moves above the detector $\left(x_{e}<-1 \mathrm{~m}\right)$, transmittance decreases exponentially with $x_{e}$. We find an exponential decay constant of $1.6 \mathrm{~m}^{-1}$ for conservative scattering and green light and $2.4 \mathrm{~m}^{-1}$ for red light. The rate of change of transmittance at $x_{e}=0$ depends on the absorption coefficient, resulting in a spatially varying spectral composition under the slab (i.e., $T_{0.5} / T_{0.05}$ depends on separation of edge and detector). The further the detector is located under the obstruction, the smaller the ratio of transmittance of red light to green light becomes, i.e. it is not only darker but also greener. At approximately $x_{e}=0.5 \mathrm{~m}$, the ratio assumes its maximum as the light is slightly redder ( $8 \%$ ) than in the far-field limit at $x_{e}>3 \mathrm{~m}$ and at $x_{e}=0$. At $x_{e}=1 \mathrm{~m}$ the difference to the far field limit is $3 \%$.

The transmittance from Monte Carlo simulations for the conservative case, $T_{\mathrm{MC}}$, is compared with three simple edge functions in Fig. 14. A linear function

$T_{\text {lin }}=\frac{T_{\infty}}{2}+a_{\text {lin }} x_{e}$,

was chosen for its geometric simplicity, a double-exponential function,

$T_{\exp }=\left\{\begin{array}{cc}T_{\infty}\left[1-\frac{1}{2} \exp \left(-a_{\exp } x_{e}\right)\right] & x_{e} \geq 0 \\ T_{\infty} \frac{1}{2} \exp \left(-a_{\exp }\left|x_{e}\right|\right) & x_{e}<0\end{array}\right.$,

because $T\left(x_{e}\right)$ depends on $x_{e}$ exponentially far from the edges, and an error function

$T_{\text {erf }}=\frac{T_{\infty}}{2}\left[1+\operatorname{erf}\left(a_{e r f} x_{e}\right)\right]$

which it has been used as empirical fit (Ehn et al., 2011).

In Eqs. (8) to (10), $T_{\infty}$ and $a$ are coefficients found from least square fitting. The linear Eq. (8) was fitted to data in $-1 \mathrm{~m}<x_{e}<1 \mathrm{~m}$, since this turned out to be the maximum range of utility of a linear approximation $\left(T_{\infty}=0.54, a_{\text {lin }}=0.22 \mathrm{~m}^{-1}\right)$. The error is $\pm 8 \%$ for $-0.9 \mathrm{~m}<x_{e}<1.3 \mathrm{~m}$. The error function in Eq. (10) was fitted to data from $-3 \mathrm{~m}<x_{e}<5 \mathrm{~m}\left(T_{\infty}=0.54, a_{e r f}=0.86 \mathrm{~m}^{-1}\right)$, resulting in an error of $\pm 5 \%$ for $-1 \mathrm{~m}<x_{e}<5 \mathrm{~m}$. For $x_{e}<-1 \mathrm{~m}$, the estimate based on the error function systematically overestimates extinction, reaching an order of magnitude by $x_{e}=-3 \mathrm{~m}$. Fitting the logarithm of transmittances (not shown) in order to reduce the 


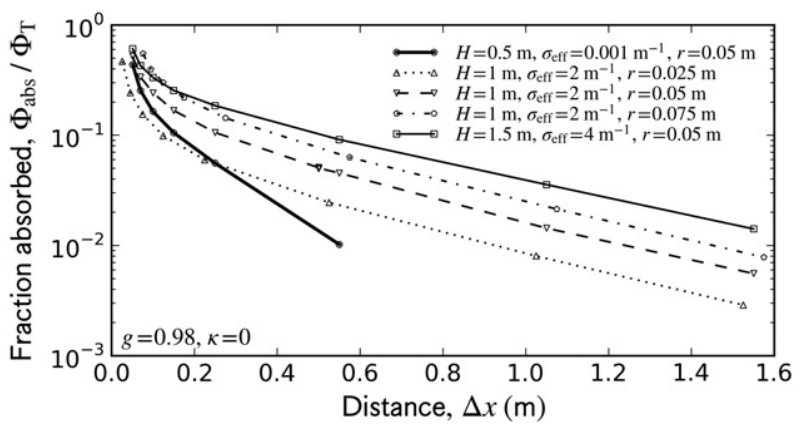

Fig. 15. Fraction of light parcels absorbed by a perfectly absorbing cylinder of radius $r$ penetrating the ice at distance $\Delta x$ from the detector. The slab of thickness $H$ is conservatively scattering with Henyey-Greenstein asymmetry parameter $g=0.98$ and effective scattering coefficient $\sigma_{\text {eff }}=\sigma(1-g)$.

error for $x_{e}<-1 \mathrm{~m}$ leads to vastly increased errors closer to the edge (errors exceeding 20\%) while still producing 50\% error at $x_{e}=-3 \mathrm{~m}$. The logarithm of the exponential function in Eq. (9) was fitted to the logarithm of transmittance model data in $-3 \mathrm{~m}<x_{e}<5 \mathrm{~m}\left(T_{\infty}=0.55\right.$, $a_{\text {exp }}=1.52 \mathrm{~m}^{-1}$ ), resulting in errors $\pm 8 \%$ for $-3 \mathrm{~m}<x_{e}<5 \mathrm{~m}$. Fitting the exponential function linearly to the data decreased performance at $x_{e}<-1 \mathrm{~m}$, leading to an overestimate of transmittance by an order of magnitude at $x_{e}=-3 \mathrm{~m}$. The fitting procedure was repeated for data from anisotropic simulations shown in Fig. 6c. While the coefficients were slightly different $\left(a_{\text {lin }}=0.23 \mathrm{~m}^{-1}, \quad a_{\text {exp }}=1.95 \mathrm{~m}^{-1}\right.$, $a_{\text {erf }}=1.09 \mathrm{~m}^{-1}$ ), the plot of transmittance ratios and errors were similar (not shown) with the most obvious feature being a worst fit of the linear function. In particular, the transmittance, $T_{\mathrm{Mc}}$, also decreased exponentially for $x_{e}<-1 \mathrm{~m}$.

\subsubsection{Cylindrical obstruction}

We aimed to determine an upper bound on the potential influence of a cylindrical perturbation penetrating the slab by considering a perfectly absorbing cylinder, e.g. a vertical pole penetrating the ice. How is the flux measured by the detector affected by proximity and size of an absorbing cylinder penetrating the ice? Using conservative scattering based on the Henyey-Greenstein phase function with $g=0.98$, and $n=1.31$, the answer is summarized in Fig. 15 for ice thickness between $H=0.5$ and $1.5 \mathrm{~m}$, cylinder radii from $r=0.025$ to $0.075 \mathrm{~m}$ (i.e., typical radii of ice augers and core barrels), and effective scattering coefficients $\sigma(1-g)=0.001,2$, and $4 \mathrm{~m}^{-1}$. The

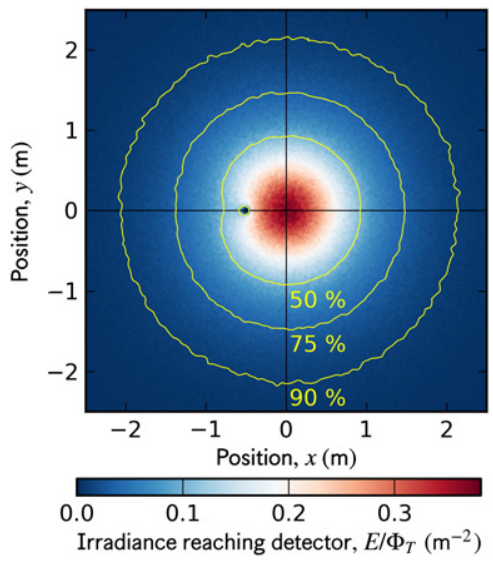

Fig. 16. Surface distribution of the areal density of the flux $\left(E / \Phi_{\mathrm{T}}\right)$ reaching a detector located beneath the origin of a $1 \mathrm{~m}$-thick, conservatively scattering slab with $\sigma(1-g)=$ $2 \mathrm{~m}^{-1}$. Diffuse illumination with absorbing cylinder (radius $r=0.05 \mathrm{~m}$ ) penetrating the slab at $x=-0.5 \mathrm{~m}$ (small dark spot). Henyey-Greenstein phase function with asymmetry parameter $g=0.98$. Contour lines show the perimeter containing $f=50,75$, and $90 \%$ of the flux registered at the detector, respectively. separation between the center of the cylinder and the detector was varied from $\Delta x=r+0.001 \mathrm{~m}$ to $\Delta x=r+1.5 \mathrm{~m}$. In all cases, absorption, i.e. the influence of the cylindrical perturbation, decreased with increasing separation $\Delta x$. The case of effective scattering coefficient $\sigma(1-g)=0.001 \mathrm{~m}^{-1}$ corresponds to essentially clear ice and shows the least amount of absorption, $\Phi_{\mathrm{abs}}$, compared to the undisturbed flux, $\Phi_{\mathrm{T}}$, of the cases with same radius $r$, while absorption is largest in the optically thickest slab. Less than $1 \%$ of the flux is absorbed by a cylinder of radius $r=0.025 \mathrm{~m}$ further than $\Delta x=1 \mathrm{~m}$ from the detector. Fig. 16 shows the irradiance incident at the surface for a cylinder of $r=0.05 \mathrm{~m}$, placed $0.5 \mathrm{~m}$ from the detector. From the point of view of the detector, irradiance is slightly reduced on the far side of the cylinder with respect to the pattern in Fig. 6a.

\section{Discussion}

We performed Monte Carlo simulations in order to obtain upper estimates of light spread in sea ice. Simulations were performed for the isotropic phase function $(g=0)$ and strongly forward-peaked Henyey-Greenstein phase function with $g=0.98$. While we found almost identical patterns of light diffusion in diffusely illuminated optically thick slabs (i.e., sea ice), we saw systematic differences in optically thin slabs (Figs. 9 through 11) and under direct illumination (Figs. 7 and 8). Since the effective phase function of multiple scattering approximates an isotropic pattern, we should expect that results from a strongly forward-peaked phase function assimilate results from an isotropic phase function after a large number of scattering events took place, i.e. in optically thick ice rather than in optically thin ice. Conversely, if incident light is directional, parcels will travel further before their first potential and potentially significant deflection in the case of isotropic scattering. This is because the density of scattering is lower (i.e., $\sigma$ is smaller) with $g=0$, as we applied the scaling relationship $\sigma(1-g)=$ const. Hence, while the concept of effective scattering coefficient may lead to useful conclusions in particular for horizontally averaged quantities (e.g., van de Hulst, 1980), limitations to its applicability exist for beam patterns in particular in optically thin slabs and under directional illumination.

For illumination of the ice by direct sunlight we analyzed the path of those light parcels that ultimately reach the detector (constituting flux $\Phi_{\mathrm{T}}$ ). We showed that the highest areal density of this flux, i.e. irradiance $E$, is shifted toward the sun. The lateral shift is up to 0.7 optical lengths $\left([\sigma(1-g)]^{-1}\right.$, Fig. 7) at the surface, which makes this effect insignificant for most applications in the presence of snow, a surface scattering layer, or granular ice (Table 2). Further, the shift decreases with increasing optical thickness and will be insignificant for ice of optical thickness below 1 (Fig. 8). However, this effect will be significant in ponded ice where 0.7 optical lengths can be expected to translate to $1 \mathrm{~m}$ or more (Table 2 ).

We found in optically thick slabs that light does not retain a significant amount of information about its conditions of incidence on the slab beyond dimensionless depth of $z \sigma(1-g) \approx-1.5$ (Fig. 8). At an equivalent scattering coefficient $\sigma(1-g)$ of $200 \mathrm{~m}^{-1}$ for snow, this means that a snow cover of $0.01 \mathrm{~m}$ depth would be sufficient to turn the propagation of light from directional into diffuse. A granular, drained surface layer in sea ice will have a similar effect. Hence, in the presence of a sufficiently thick and highly scattering surface layer it would be appropriate to treat light penetrating the interior ice as diffuse. In particular, lateral deflections shown in Fig. 7 would be much reduced.

We made an interesting observation tied to the index of refraction $n>1$ : for optically very thin ice and diffuse illumination, and in particular under isotropic scattering, the radial distribution of the flux reaching the detector is different from the case shown in Fig. 6a. I.e., the highest sensitivity to ice conditions is found away from the center toward larger radial distances (Fig. $11 \mathrm{~b}$ for $H \sigma<0.25$ ). This happens because scattering is rare and transmission is well described by 
geometric optics, resulting in the highest radiance inside the slab at the angle of total internal reflection (Snell's law). Since this phenomenon is tied to optically thin slabs it may be encountered more frequently in frozen freshwater bodies and in nilas.

We saw that absorption acts to reduce the effective lateral spread of light (Figs. 6d and 12). Absorption is an exponential function of the path length of light parcels, and the path length tends to be longest for parcels incident on the slab furthest from the detector. As a result, attenuation will be most pronounced for parcels at large radial distances, $R$, narrowing the spread of the flux reaching the detector. For the same reason, narrowing of the effective spread of light will take place in the presence of absorbing layers within the ice, e.g. sediment, detritus, or algae layers.

Optical anisotropy also narrows the spread of the flux from surface to detector, albeit for different reasons. In this case, vertically traveling parcels are simply less likely to get scattered in different directions than horizontally traveling parcels. While we saw in an example calculation that the effect of optical anisotropy on beam spread is significant, i.e. reducing radii at the surface by $30 \%$ (Fig. 6c), the appropriate ratio of scattering parameters to use is currently poorly constraint. Characterizing the anisotropic properties of sea ice is made difficult by the fact that the pore volume and morphology are functions of growth and melt history, bulk salinity and temperature (e.g., Light et al., 2003b; Petrich et al., 2006; Pringle et al., 2009). For our case study of a $1 \mathrm{~m}$ thick, conservatively scattering slab with (isotropic) scattering coefficient $\sigma(1-g)=2 \mathrm{~m}^{-1}$ we found that pairs of anisotropic scattering parameters $\sigma_{\mathrm{v}}(1-g)$ and $\sigma_{\mathrm{h}}(1-g)$ resulting in the same transmittance were 1.19 and $2.38 \mathrm{~m}^{-1}$, and 0.87 and $2.61 \mathrm{~m}^{-1}$, for $\sigma_{\mathrm{h}} / \sigma_{\mathrm{v}}=2$ and 3 respectively. Hence, scattering coefficients derived from transmittance measurements that were interpreted with an optical isotropic model would have been around 20\% lower and 40\% higher than the horizontal and vertical scattering coefficients assuming an optically anisotropic slab with $\sigma_{\mathrm{h}} / \sigma_{\mathrm{v}}=2$.

Example calculations of beam spread in $1 \mathrm{~m}$ thick ice beneath a semi-infinite surface obstruction show that transmittance measurements may be affected noticeably within approximately $1 \mathrm{~m}$ of the edge. The effect is mostly that of darkening but a small shift in spectral composition may be observable (Fig. 13). Apart from the $1 \mathrm{~m}$ closest to the edge, the flux measured under the obstruction decays exponentially with distance from the edge (Fig. 14). This case study can be interpreted in a more general way. For conservatively scattering and optically reasonably thick ice $(H \sigma(1-g)>1)$, we see from Fig. 9 that the beam spread pattern at the surface relative to ice thickness (i.e., $R / H$ ) does not vary much with optical thickness. In first approximation we can assume that the lateral spread of light scales linearly with ice thickness. Then darkening by a semi-infinite surface obstruction is very well described $(<5 \%)$ by an error function except under the obstruction further from the edge than the ice is thick $\left(x_{e}<-H\right)$. For accuracy further beneath the surface obstruction a double-exponential function may be used, although it is slightly less accurate near the edge. These functions were also found to describe darkening well in simulations with an optically anisotropic slab. Ehn et al. (2011) used an error function to describe darkening at the edge between melt ponds and white ice, finding coefficients $0.6 \mathrm{~m}^{-1} \leq a_{\mathrm{erf}} \leq 1 \mathrm{~m}^{-1}$, i.e. a range overlapping with our two case studies $\left(0.8 \mathrm{~m}^{-1} \leq a_{\mathrm{erf}} \leq 1.1 \mathrm{~m}^{-1}\right)$.

Using a three-dimensional geometry of an absorbing cylinder penetrating $1 \mathrm{~m}$ thick ice we found that darkening at the detector due to absorption by the cylinder (for radii around $r=0.025 \mathrm{~m}$ ) is less than $1 \%$ at distances of $1 \mathrm{~m}$. Darkening will be less under direct illumination (i.e., clear skies), if ice is optically anisotropic (likely the case) or in the presence of ice absorption or other absorbing substances in the ice interior (also likely), and if scattering coefficients are lower than assumed (Table 2). The cylinder could be the pole of a measurement set-up used to suspend a sensor beneath the ice (e.g., Fig. 5). In this case, cylinder radii of $0.05 \mathrm{~m}$ and $0.075 \mathrm{~m}$ are probably unrealistically large. Also, using white poles to penetrate the ice would reduce the darkening by the cylinder. However, we did not consider shading from instruments mounted above the ice. Nicolaus et al. (2010b) calculated the effect of shading of instrument support on albedo measurements finding that corrections in the range of $1 \%$ to $10 \%$ were appropriate.

Localized, small obstructions at the surface have a negligible influence on transmittance. For example, from Fig. 11a, a $0.1 \mathrm{~m}^{2}$ obstruction at the surface of $1 \mathrm{~m}$ thick ice at $1 \mathrm{~m}$ distance from the detector will reduce the flux at the detector by less than $1 \%$.

Point measurements of transmittance under sea ice measure spatially weighted, average properties of ice interior and surface (Figs. 9 and 10 ). We found that the sensitivity of a measurement to surface disturbances decreases exponentially with radial distance from the detector, $R$, for $R>H$ (Fig. 11a). For $R<H$, the sensitivity is less dependent on $R$, in particular for $R / H<0.25$. For example, we expect that in $1 \mathrm{~m}$ thick ice a cover of $0.01 \mathrm{~m}^{2}$ placed on the surface at $1 \mathrm{~m}$ distance from the detector will affect the measured flux by $0.1 \%$, almost independently of scattering properties (Fig. 11).

Regarding the question whether 3-dimensional light propagation through sea ice can be modeled with 1-dimensional (1D) light transfer models we refer to Fig. 9. Light transfer can, in principle, be described by 1D models if ice can be described as a stack of homogeneous slabs of laterally infinite extent, each with its own optical properties and thickness (e.g., Grenfell, 1991). Hence, we need to define a homogeneous slab. For the purpose of transmittance measurements a slab is homogeneous if the transmittance measurement is independent of the location of measurement (i.e., it is invariant to translation of the detector). Hence, the slab has to be laterally large enough for edge effects to be negligible, and any imperfections inside the slab have to be small enough to be undetectable beneath the slab. Let us assume that variations of transmittance measurements of $10 \%$ are deemed tolerable. From Fig. 9 we find immediately that $90 \%$ of the detected light enters the slab within a radius of twice the slab thickness $(R=2 H)$ around the measurement (for conservatively scattering slab, i.e. in reality the radius will likely be less). Hence, by requiring homogeneity over this range we can be reasonably certain that almost all light parcels (i.e., 90\%) will have traversed ice with the same optical properties. Small-scale imperfections are unavoidable in sea ice as they are the reason for scattering in the first place. From Fig. 11 we note that the largest areal density of the flux of light parcels reaching the detector is directly above the detector ( $R=0$, under diffuse illumination), and from Fig. 9 we note that $10 \%$ of the flux passes through a circle of radius $R=0.3 H$ (for a conservatively scattering slab, i.e. including absorption a more conservative estimate would be $R=0.2 H$ ). As long as imperfections are randomly distributed, this will be the upper limit on the size of surface imperfections allowable. We see from Fig. 10 that the circle most traversed by light parcels narrows with depth as we approach the detector, leading to more stringent requirements on imperfections inside the ice. We suggest that ice can be modeled with a $1 \mathrm{D}$ approach if it is homogeneous within a radius of $2 \mathrm{H}$ around the measurement, where homogeneous means that significant, randomly distributed surface imperfections are of radius smaller than $0.2 \mathrm{H}$.

\section{Summary and conclusion}

A three-dimensional Monte Carlo model of light propagation through a homogeneous slab of sea ice was used to derive upper bounds on light spread. Due to the effect of multiple scattering on the net phase function, the phase function has a small (direct light) or negligible effect (diffuse light) on the mean path of light transmitted through optically thick ice. Anisotropic optical properties were considered and found to narrow the light spread. 
We find that under direct sunlight the path of light may be deflected toward the sun by the effective scattering length (i.e., the reciprocal of the effective scattering coefficient), which can be as much as $1 \mathrm{~m}$ and more in ponded ice (cf. Table 2). The net path of light becomes independent of incident light under direct illumination at dimensionless depths $z \sigma(1-g)<-1.5$, implying that a few centimeters of snow above sea ice are sufficient to turn direct light into diffuse light.

We argue that sea ice can be treated with one-dimensional radiative transfer models, and that constraints placed on ice conditions depend on the accuracy of desired measurements. To obtain $10 \%$ measurement accuracy we find as upper limit that ice around the detector has to be laterally homogeneous over a range of twice the ice thickness, provided that irregularities in ice optical properties are of radius smaller than 0.2 times ice thickness. More stringent requirements on patch size apply to irregularities at depth, closer to the detector. As a rule, in the absence of absorption, $10 \%$ and $90 \%$ of the flux detected under optically thick ice are incident on the surface within a radius of less than $R=0.3 H$ and $R=2 H$, respectively (Fig. 9). The upper half of the ice interior is probed over approximately the same lateral range as the surface while this range narrows toward the bottom of the ice (Fig. 10). Absorption and optical anisotropy narrow the spatial range of sensitivity (Figs. 6 and 12). While the strength of this effect depends on ice thickness and scattering properties, a ballpark figure for light at $700 \mathrm{~nm}$ would be a range reduction to approximately $80 \%$ of its value in the absence of absorption. The presence of substances such as colored dissolved organic matter (CDOM), detritus, and layers of sediment or ice algae will increase absorption and narrow the range further. The suggested anisotropy of ice as a scattering medium would further narrow beam spread (Haines et al., 1997).

Using the assumption of conservative scattering allowed us to derive relationships that scale with ice thickness and scattering coefficient. It also allowed us to determine upper bounds for expected errors in transmittance measurements. While we were investigating beam spread patterns to determine maximum errors due to inhomogeneities in the ice and at the surface, uncertainties in scattering and absorption coefficients (e.g., colored dissolved organic matter, algae, detritus, sediment) may outweigh by far systematic errors introduced from surface perturbations or instruments deployed inside the ice. Anisotropic optical properties of ice are currently not well characterized but may be significant. Identified perturbations in the ice can be accounted for in post processing, and we gave guidance to estimating their significance.

The present work should address the most pressing questions regarding the length scales to define sea ice as homogeneous and beam spread inside ice. However, there is no limit to the number of relevant case studies and geometries that could be considered. We considered circular geometry, directional illumination, semi-infinite perturbances at the surface, and cylinders penetrating the ice. Also light measurements at distance from the ice-ocean interface should be considered. Multi-layer simulations could be performed but would be even more valuable after a field campaign when specific ice conditions are known.

\section{Acknowledgments}

This study was motivated by questions raised in peer review regarding the possibility of modeling transmittance in sea ice with one dimensional light transfer models. Financial support for CP was provided by a proposal preparation grant of the Geophysical Institute of the University of Alaska Fairbanks. Support for RG was provided by National Science Foundation (NSF) award \#0732767. Helpful comments of Dr. Bonnie Light and Christian Katlein in the preparation of this manuscript are gratefully acknowledged. CP owes his interest in light scattering to inspiring remarks of Dr. Joe Trodahl. The constructive comments of two anonymous reviewers helped to improve the presentation.

\section{References}

Bohren, C.F., 1987. Multiple scattering of light and some of its observable consequences. American Journal of Physics 55, 524-533.

Briegleb, B.P., Light, B., 2007. A delta-Eddington multiple scattering parameterization for solar radiation in the sea ice component of the Community Climate System Model. NCAR Technical Note TN-472. National Center for Atmospheric Research (NCAR), Boulder, CO. 100 pp.

Buckley, R.G., Trodahl, H.J., 1987. Scattering and absorption of visible light by sea ice. Nature 326, 867-869.

Chandrasekhar, S., 1960. Radiative Transfer. Dover, New York, 393 pp.

Ehn, J.K., Mundy, C.J., Barber, D.G., Hop, H., Rossnagel, A., Stewart, J., 2011. Impact of horizontal spreading on light propagation in melt pond covered seasonal sea ice in the Canadian Arctic. Journal of Geophysical Research 116, C00G02. doi:10.1029/2010JC006908.

Gradinger, R., 2009. Sea-ice algae: major contributors to primary production and algal biomass in the Chukchi and Beaufort Seas during May/June 2002. Deep Sea Research Part II: Topical Studies in Oceanography 56, 1201-1212.

Gradinger, R.R., Kaufman, M.R., Bluhm, B.A., 2009. Pivotal role of sea ice sediments in the seasonal development of near-shore Arctic fast ice biota. Marine Ecology Progress Series 394, 49-63.

Grenfell, T.C., 1991. A radiative transfer model for sea ice with vertical structure variations. Journal of Geophysical Research 96 (C9), 16,991-17,001.

Grenfell, T.C., Warren, S.G., 1999. Representation of a nonspherical ice particle by a collection of independent spheres for scattering and absorption of radiation. Journal of Geophysical Research 104 (D24), 31,697-31,709.

Haines, E.M., Buckley, R.G., Trodahl, H.J., 1997. Determination of the depth-dependent scattering coefficient in sea ice. Journal of Geophysical Research 102 (C1), 1141-1151.

Henyey, L.G., Greenstein, J.L., 1941. Diffuse radiation in the galaxy. The Astrophysical Journal 93, 70-83.

Light, B., Maykut, G.A., Grenfell, T.C., 2003a. Effects of temperature on the microstructure of first-year Arctic sea ice. Journal of Geophysical Research 108 (C2), 3051. doi:10.1029/2001JC000887.

Light, B., Maykut, G.A., Grenfell, T.C., 2003b. A two-dimensional Monte Carlo model of radiative transfer in sea ice. Journal of Geophysical Research 108 (C7), 3219. doi:10.1029/2002JC001513.

Light, B., Maykut, G.A., Grenfell, T.C., 2004. A temperature-dependent, structural-optical model of first-year sea ice. Journal of Geophysical Research 109, C06013. doi:10.1029/2003JC002164.

Light, B., Grenfell, T.C., Perovich, D.K., 2008. Transmission and absorption of solar radiation by Arctic sea ice during the melt season. Journal of Geophysical Research 113, C03023. doi:10.1029/2006JC003977.

Maffione, R.A., Voss, J.M., Mobley, C.D., 1998. Theory and measurements of the complete beam spread function of sea ice. Limnology and Oceanography 43 (1), 34-43.

Matsumoto, M., Nishimura, T., 1998. Mersenne Twister: a 623-dimensionally equidistributed uniform pseudorandom number generator. ACM Transactions on Modeling and Computer Simulation 8 (1), 3-30.

Mobley, C.D., Cota, G.F., Grenfell, T.C., Maffione, R.A., Pegau, W.S., Perovich, D.K., 1998. Modeling light propagation in sea ice. IEEE Transactions on Geoscience and Remote Sensing 36, 1743-1749.

Nicolaus, M., Gerland, S., Hudson, S.R., Hanson, S., Haapala, J., Perovich, D.K., 2010a. Seasonality of spectral albedo and transmittance as observed in the Arctic Transpolar Drift in 2007. Journal of Geophysical Research 115, C11011. doi:10.1029/ 2009JC006074.

Nicolaus, M., Hudson, S.R., Gerland, S., Munderloh, K., 2010b. A modern concept for autonomous and continuous measurements of spectral albedo and transmittance of sea ice. Cold Regions Science and Technology 62 (1), 14-28.

Pegau, W.S., Zaneveld, J.R.V., 2000. Field measurements of in-ice radiance. Cold Regions Science and Technology 31, 33-46.

Perovich, D.K., 1990. Theoretical estimates of light reflection and transmission by spatially complex and temporally varying sea ice covers. Journal of Geophysical Research 95, 9557-9567.

Petrich, C., Langhorne, P.J., Sun, Z.F., 2006. Modelling the interrelationships between permeability, effective porosity and total porosity in sea ice. Cold Regions Science and Technology 44, 131-144.

Piskozub, J., McKee, D., 2011. Effective scattering phase functions for the multiple scattering regime. Optics Express 19 (5), 4786-4794.

Pringle, D.J., Miner, J.E., Eicken, H., Golden, K.M., 2009. Pore space percolation in sea ice single crystals. Journal of Geophysical Research 114, C12017. doi:10.1029/ 2008JC005145.

Segelstein, D. J. (1981), The complex refractive index of water. Thesis (M.S.), University of Missouri-Kansas City, 167 pp.

Sobouti, Y., 1963. Chandrasekhar's X-, Y-, and related functions. Astrophysical Journal Supplement 7, 411-560.

Thomas, G.E., Stamnes, K., 1999. Radiative Transfer in the Atmosphere and Ocean. Cambridge University Press, New York. 517 pp.

Van de Hulst, H.C., 1980. Multiple Light Scattering: Tables, Formulas, and Applications, Vol. 2. Academic Press, New York.

Warren, S.G., Brandt, R.E., 2008. Optical constants of ice from the ultraviolet to the microwave: a revised compilation. Journal of Geophysical Research 113. doi:10.1029/ 2007JD009744

Zege, E.P., Ivanov, A.P., Katsev, I.L., 1991. Image Transfer Through a Scattering Medium. Springer Verlag, New York. 349 pp. 